\title{
Н.Н. Трофименко
}

\section{О ЛИНЕЙНЫХ ГОМЕОМОРФИЗМАХ ПРОСТРАНСТВ НЕПРЕРЫВНЫХ ФУНКЦИЙ НА «ДЛИННЫХ ПРЯМЫХ»}

\begin{abstract}
Доказывается, что для начального регулярного несчетного ординала $\tau$ и произвольных начальных ординалов $\alpha, \beta, \alpha<\beta \leq \tau$, пространства непрерывных функций $C_{p}\left(L_{\tau \cdot \alpha}\right)$ и $C_{p}\left(L_{\tau \cdot \beta}\right)$, заданные на «длинных прямых» $L_{\tau \cdot \alpha}$ и $L_{\tau \cdot \beta}$, не являются линейно гомеоморфными.
\end{abstract}

Ключевые слова: «длинные прямые», линейные гомеоморфизмы, сопряженное пространство, ординаль, начальный ординал, регулярный ординал, топология поточечной сходимости, компактность.

Рассматриваются пространства непрерывных функций $C\left(L_{\alpha}\right)$, заданные на «длинных прямых» $L_{\alpha}$, где $\alpha-$ произвольный ординал. Пространства непрерывных функций $C\left(L_{\alpha}\right)$ наделяются топологией поточечной сходимости и обозначаются $C_{p}\left(L_{\alpha}\right)$. «Длинные прямые» - это частный случай линейно упорядоченных топологических пространств. Пространства непрерывных функций, заданные на линейно упорядоченных топологических пространствах, и их изоморфная классификация рассматривались во многих работах, например в [2-6].

Определение 1 . Пусть $\alpha$ - произвольный ординал. Рассмотрим линейное упорядочение < на множестве $L_{\alpha}=[1, \alpha] \times[0,1)$, определенное так: $\left(\mu_{1}, t_{1}\right)<\left(\mu_{2}, t_{2}\right)$, если $\mu_{1}<\mu_{2}$ или $\mu_{1}=\mu_{2}$ и $t_{1}<t_{2}$. Будем называть «длинной прямой» множество $L_{\alpha}$ с топологией, порожденной линейным упорядочением $<$.

Заметим, что топологическое пространство $L_{\alpha}$ является компактным.

Будем говорить, что точка $x=(\xi, t) \in L_{\alpha}$ конфинальна ординалу $\eta$, если в интервале $((1,0),(\xi, t))$ существует конфинальное подмножество, подобное отрезку ординалов $[0, \eta)$.

Напомним, что ординал $\alpha$ называется начальным, если $\alpha$ - наименьший среди всех ординалов $\lambda$, таких, что $|\lambda|=|\alpha|$. Начальный ординал $\alpha$ называется регулярным, если не существует $\lambda<\alpha$, конфинального $\alpha$.

Основным результатом работы является следующая теорема.

Теорема 1. Пусть $\tau$ - регулярный начальный несчетный ординал и $\alpha, \beta$ начальные ординалы, такие, что $\alpha<\beta \leq \tau$. Тогда пространства $C_{p}\left(L_{\tau \cdot \alpha}\right)$ и $C_{p}\left(L_{\tau \cdot \beta}\right)$ не являются линейно гомеоморфными.

Для доказательства этой теоремы нам понадобится следующие вспомогательные утверждения. 
Предложение 1. Пусть $\alpha \leq \tau$. Если точка $x \in L_{\tau \cdot \alpha}$ конфинальна $\tau$, то $x=(\tau \cdot(\gamma+1), 0), 0 \leq \gamma<\alpha$, или $x=(\tau \cdot \tau, 0)$, если $\alpha=\tau$.

Доказательство. Пусть $\alpha<\tau$ и $x=(\xi, t)$. Если $0<t<1$ или $t=0$, а $\xi-$ непредельный ординал, то точка $x$ конфинальна $\omega$. Рассмотрим точки вида $x=(\xi, 0)$, где $\xi-$ предельный ординал. Согласно [8], ординал $\xi$ можно представить в виде $\xi=\tau \cdot \eta+\rho$, где $0 \leq \rho<\tau, 0 \leq \eta<\alpha$ или $\xi=\tau \cdot \alpha$. Если $\rho>0$, то ординал $\xi$ конфинален ординалу $\rho<\tau$ и, следовательно, $x$ конфинально ординалу $\rho$. Если $\rho=0$ и $\xi=\tau \cdot \eta$, где $\eta-$ предельный ординал, то $\xi$ конфинален $\eta$, где $\eta \leq \alpha<\tau$. Если $\eta-$ непредельный ординал, т.е. $\eta=\gamma+1$, то точка $x=(\tau \cdot \gamma+\tau, 0)$ конфинальна $\tau$. Итак, множество точек, конфинальных $\tau$, это в точности все точки вида $(\tau \cdot(\gamma+1), 0), 0 \leq \gamma<\alpha$.

Пусть теперь $\alpha=\tau$. Аналогично доказывается, что если точка $x<(\tau \cdot \tau, 0)$ и конфинальна $\tau$, то $x=(\tau \cdot(\gamma+1), 0)$. Но в этом случае точка $x=(\tau \cdot \tau, 0)$ также конфинальна $\tau$.

Положим

$$
\Gamma_{\alpha}=\{(\tau \cdot(\gamma+1), 0), \gamma \in[0, \alpha)\} \text {, если } \alpha<\tau, \Gamma_{\tau}=\{(\tau \cdot(\gamma+1), 0), \gamma \in[0, \tau)\} \bigcup\{(\tau \cdot \tau, 0)\},
$$

и

$$
c_{0}\left(\Gamma_{\alpha}\right)=\left\{x \in C_{p}\left(\Gamma_{\alpha}\right):\left\{t \in \Gamma_{\alpha} \text {, таких, что }|x(t)| \geq \varepsilon\right\} \text { конечно } \forall \varepsilon>0\right\} \text {. }
$$

В пространстве $\mathbb{R}^{L_{\tau \cdot \alpha}}$ рассмотрим линейное подпространство

$$
\begin{aligned}
M_{\tau \cdot \alpha} & =\left\{y \in \mathbb{R}^{L_{\tau \cdot \alpha}}: \forall\left\{f_{i}\right\}_{i \in I} \in L_{p}\left(L_{\tau \cdot \alpha}\right), \text { такого, что }|I|<|\tau|,\right. \\
& \left.\exists x \in C_{p}\left(L_{\tau \cdot \alpha}\right) \text { такой, что } f_{i}(x)=f_{i}(y) \forall i \in I\right\} .
\end{aligned}
$$

Очевидно, что все непрерывные функции на $L_{\tau \cdot \alpha}$ принадлежат $M_{\tau \cdot \alpha}$. Кроме того, пространству $M_{\tau \cdot \alpha}$ принадлежат те разрывные функции $y$, для которых любого семейства функционалов мощности меньшей $|\tau|$ недостаточно для разделения $y$ и точек пространства $C_{p}\left(L_{\tau \cdot \alpha}\right)$. Далее покажем, что пространство $M_{\tau \cdot \alpha}-$ это прямая сумма пространства $C_{p}\left(L_{\tau \cdot \alpha}\right)$ и некоторого подпространства в $\mathbb{R}^{L_{\tau \cdot \alpha}}$, линейно гомеоморфного пространству $c_{0}\left(\Gamma_{\alpha}\right)$, т.е. верно следующее утверждение.

Предложение 2. Пространство $M_{\tau \cdot \alpha}$ линейно гомеоморфно пространству $C_{p}\left(L_{\tau \cdot \alpha}\right) \times c_{0}\left(\Gamma_{\alpha}\right)$.

Доказательство. Определим отображение $T: C_{p}(L) \times c_{0}\left(\Gamma_{\alpha}\right) \rightarrow \mathbb{R}^{L_{\tau \cdot \alpha}}$ по формуле $T(x, y)=x+\tilde{y}$, где

$$
\tilde{y}(t)=\left\{\begin{array}{l}
y(t), \text { если } t \in \Gamma_{\alpha} ; \\
0, \text { если } t \in L_{\tau \cdot \alpha} \backslash \Gamma_{\alpha} .
\end{array}\right.
$$

Докажем, что отображение $T$ является инъективным. Пусть $\left(x_{1}, y_{1}\right),\left(x_{2}, y_{2}\right) \in C_{p}(L) \times c_{0}\left(\Gamma_{\alpha}\right)$. Предположим, что

$$
T\left(x_{1}, y_{1}\right)=x_{1}+\tilde{y}_{1}=x_{2}+\tilde{y}_{2}=T\left(x_{2}, y_{2}\right),
$$


т.е. $x_{1}-x_{2}=\tilde{y}_{2}-\tilde{y}_{1}$. Поскольку $\left.\left(\tilde{y}_{2}-\tilde{y}_{1}\right)\right|_{L_{\tau \cdot \alpha} \backslash \Gamma_{\alpha}}=0$, то $\left.\left(x_{1}-x_{2}\right)\right|_{L_{\tau \cdot \alpha} \backslash \Gamma_{\alpha}}=0$. Так как функция $x_{1}-x_{2}$ является непрерывной, а множество $L_{\tau \cdot \alpha} \backslash \Gamma_{\alpha}$ является всюду плотным в $L_{\tau \cdot \alpha}$, то $x_{1}-x_{2} \equiv 0$. Следовательно, $\tilde{y}_{2}-\tilde{y}_{1} \equiv 0$, т.е. $\left(x_{1}, y_{1}\right)=\left(x_{2}, y_{2}\right)$.

Докажем, что $T\left(C_{p}(L) \times c_{0}\left(\Gamma_{\alpha}\right)\right)=M_{\omega_{\tau} \cdot \omega_{\alpha}}$. Пусть $(x, y) \in C_{p}(L) \times c_{0}\left(\Gamma_{\alpha}\right)$. Рассмотрим множество функционалов $\left\{f_{i}\right\}_{i \in I} \in L_{p}\left(L_{\tau \cdot \alpha}\right)$, для которого $|I|<|\tau|$. Так как для любого $i \in I \quad\left|\operatorname{supp} f_{i}\right|<\aleph_{0}$, то $\left|\bigcup_{i \in I} \operatorname{supp} f_{i}\right|<|\tau|$. Поскольку ординал $\tau$ неконфинален никакому ординалу, меньшему чем $\tau$, то для каждого $\gamma<\alpha$ существует ординал $\delta_{\gamma}, \tau \cdot \gamma<\delta_{\gamma}<\tau \cdot(\gamma+1)$, такой, что

$$
\left(\left(\delta_{\gamma}, 0\right),(\tau \cdot(\gamma+1), 0)\right) \cap\left(\bigcup_{i \in I} \operatorname{supp} f_{i}\right)=\varnothing .
$$

Рассмотрим функцию

$$
z(t)=\left\{\begin{array}{l}
y(\tau \cdot(\gamma+1), 0), \text { если } t \in\left(\left(\delta_{\gamma}, 0\right),(\tau \cdot(\gamma+1), 0)\right] ; \\
0, \text { в остальных случаях. }
\end{array}\right.
$$

Нетрудно видеть, что функция $z$, а значит, и $x+z$ являются непрерывными на $L_{\tau \cdot \alpha}$. Поскольку функции $x+z$ и $x+\tilde{y}$ отличаются только на множестве $\bigcup_{\gamma \in\left[0, \omega_{\alpha}\right]}\left(\delta_{\gamma}, 0,(\tau \cdot(\gamma+1), 0)\right.$, то $f_{i}(x+z)=f_{i}(x+\tilde{y})$ для любого $i \in I$. Следовательно, $T(x, y)=x+\tilde{y} \in M_{\tau \cdot \alpha}$.

Пусть теперь $z \in M_{\tau \cdot \alpha}$. Покажем, что функция $z$ может иметь разрыв только в точках из множества $\Gamma_{\alpha}$. Действительно, если точка разрыва $t_{0} \notin \Gamma_{\alpha}$, то существует множество $A,|A|<|\tau|$, такое, что $\sup \mu=t_{0}$. Рассмотрим множество функ$\mu \in A$

ционалов $\left\{\delta_{\mu}-\delta_{t_{0}}\right\}_{\mu \in A}$. Если функция z имеет разрыв в точке $t_{0}$, то существует $\varepsilon_{0}>0$, такое, что для любой окрестности $U_{t_{0}}$ найдется $\mu \in A \cap U_{t_{0}}$, для которого $\left|z(\mu)-z\left(t_{0}\right)\right| \geq \varepsilon_{0}$, то есть $\left|\left(\delta_{\mu}-\delta_{t_{0}}\right)(z)\right| \geq \varepsilon_{0}$. Если же функция $x \in C_{p}\left(L_{\tau \cdot \alpha}\right)$, то существует окрестность $V_{t_{0}}$, такая, что для любого $\mu \in V_{t_{0}}$

$$
\left|x(\mu)-x\left(t_{0}\right)\right|=\left|\left(\delta_{\mu}-\delta_{t_{0}}\right)(x)\right|<\varepsilon_{0} .
$$

Это противоречит тому, что $z \in M_{\tau \cdot \alpha}$. Таким образом, любая функция $z \in M_{\tau \cdot \alpha}$ непрерывна на множестве $L_{\tau \cdot \alpha} \backslash \Gamma_{\alpha}$.

Рассмотрим функцию $\left.z\right|_{((\tau \cdot \gamma, 0),(\tau \cdot(\gamma+1), 0))}$. Поскольку точка $(\tau \cdot(\gamma+1), 0)$ не конфинальна $\omega$, то существует ординал $\eta_{\gamma} \in(\tau \cdot \gamma, \tau \cdot(\gamma+1))$, такой, что $\left.z\right|_{\left(\left(\eta_{\gamma}, 0\right),(\tau \cdot(\gamma+1), 0)\right)} \equiv z\left(\left(\eta_{\gamma}, 0\right)\right)$. Определим функцию

$$
\tilde{z}(t)=\left\{\begin{array}{l}
z(t), \text { если } t \notin \Gamma_{\alpha} ; \\
z\left(\eta_{\gamma}\right), \text { если } t=\tau \cdot(\gamma+1) .
\end{array}\right.
$$


Нетрудно видеть, что функция $\tilde{z}$ непрерывна на $L_{\tau \cdot \alpha}$ и $z-\left.\tilde{z}\right|_{L_{\tau \cdot \alpha} \backslash \Gamma_{\alpha}} \equiv 0$. Покажем, что $\left.(z-\tilde{z})\right|_{\Gamma_{\alpha}} \in c_{0}\left(\Gamma_{\alpha}\right)$. Предположим, что для некоторого $\varepsilon>0$ существует последовательность $\left\{\left(\tau \cdot\left(\gamma_{n}+1\right), 0\right)\right\}_{n=1}^{\infty} \subset \Gamma_{\alpha}$ такая, что

$$
\left|(z-\tilde{z})\left(\left(\tau \cdot\left(\gamma_{n}+1\right), 0\right)\right)\right| \geq \varepsilon .
$$

Поскольку множество $L_{\tau \cdot \alpha}$ является компактным, то для множества $\left\{\left(\tau \cdot\left(\gamma_{n}+1\right), 0\right)\right\}_{n=1}^{\infty} \subset \Gamma_{\alpha}$ существует предельная точка $x=(\xi, t)$. Так как множество $\Gamma_{\alpha}$ дискретное, то $x=(\xi, t) \notin \Gamma_{\alpha}$ и, значит, $(z-\tilde{z})(x)=0$. Так как точка $x=(\xi, t)$ является предельной для $\left\{\left(\tau \cdot\left(\gamma_{n}+1\right), 0\right)\right\}_{n=1}^{\infty} \subset \Gamma_{\alpha}$, то в силу неравенства (3) получаем, что функция $z-\tilde{z}$ является разрывной в точке $x$, что противоречит тому, что функции $z$ и $\tilde{z}$ непрерывны во всех точках $x \notin \Gamma_{\alpha}$. Следовательно,

$$
z=z+(z-\tilde{z})=T\left(\tilde{z},\left.(z-\tilde{z})\right|_{\Gamma_{\alpha}}\right) \in C_{p}\left(L_{\tau \cdot \alpha}\right) \times c_{0}\left(\Gamma_{\alpha}\right) .
$$

Таким образом, $T\left(C_{p}(L) \times c_{0}\left(\Gamma_{\alpha}\right)\right)=M_{\tau \cdot \alpha}$. Легко проверить, что отображения $T$ и $T^{-1}$ являются непрерывными. Заключаем, что пространства $M_{\tau \cdot \alpha}$ и $C_{p}\left(L_{\tau \cdot \alpha}\right) \times c_{0}\left(\Gamma_{\alpha}\right)$ являются линейно гомеоморфными.

Доказательство теоремы 1. Доказательство теоремы проведем методом от противного. Предположим, что существует линейный гомеоморфизм Ф пространства $C_{p}\left(L_{\tau \cdot \beta}\right)$ на пространство $C_{p}\left(L_{\tau \cdot \alpha}\right)$.

Поскольку пространства $C_{p}\left(L_{\tau \cdot \alpha}\right)$ и $C_{p}\left(L_{\tau \cdot \beta}\right)$ всюду плотны в пространствах $\mathbb{R}^{L_{\tau \cdot \alpha}}$ и $\mathbb{R}^{L_{\tau \cdot \beta}}$ соответственно, то линейный гомеоморфизм $\Phi$ может быть продолжен до линейного гомеоморфизма $\tilde{\Phi}$ пространства $\mathbb{R}^{L_{\tau \cdot \beta}}$ на пространство $\mathbb{R}^{L_{\tau \cdot \alpha}} \quad[1$, c. 654].

Известно [1], что сопряженным к пространствам $C_{p}\left(L_{\tau \cdot \alpha}\right)$ и $\mathbb{R}^{L_{\tau \cdot \alpha}}$ является пространство $L_{p}\left(L_{\tau \cdot \alpha}\right)$, состоящее из функционалов вида

$$
f=p_{1} \cdot \delta_{t_{1}}+p_{2} \cdot \delta_{t_{2}}+\ldots+p_{n} \cdot \delta_{t_{n}},
$$

где $p_{k} \in \mathbb{R} \backslash\{0\}$ и $\delta_{t_{k}}(x)=x\left(t_{k}\right)$ для любого $x \in C_{p}\left(L_{\tau \cdot \alpha}\right), k=1, \ldots, n$. Множество точек $\left\{t_{1}, \ldots, t_{n}\right\} \subset L_{\tau \cdot \alpha}$ называется носителем функционала $f$ и обозначается supp $f$.

Покажем, что

$$
\tilde{\Phi}\left(M_{\tau \cdot \beta}\right)=M_{\tau \cdot \alpha}
$$

Пусть функция $y \in M_{\tau \cdot \beta}$. Рассмотрим произвольное семейство функционалов $\left\{g_{i}\right\}_{i \in I} \in L_{p}\left(L_{\tau \cdot \alpha}\right)$, где $|I|<|\tau|$. По определению множества $M_{\tau \cdot \beta}$ для семейства функционалов $\left\{f_{i}\right\}_{i \in I}=\left\{\tilde{\Phi}^{*} g_{i}\right\}_{i \in I} \in L_{p}\left(L_{\tau \cdot \beta}\right)$ существует непрерывная функция 
$x \in C_{p}\left(L_{\tau \cdot \beta}\right)$, такая, что

$$
\left(\tilde{\Phi}^{*} g_{i}\right)(x)=\left(\tilde{\Phi}^{*} g_{i}\right)(y)
$$

для любого $i \in I$. Отсюда, по определению отображения $\tilde{\Phi}^{*}: L_{p}\left(L_{\tau \cdot \alpha}\right) \rightarrow L_{p}\left(L_{\tau \cdot \beta}\right)$ получаем, что

$$
g_{i}(\tilde{\Phi} x)=g_{i}(\tilde{\Phi} y)
$$

для любого $i \in I$. Поскольку функция $\tilde{\Phi} x \in C_{p}\left(L_{\tau \cdot \alpha}\right)$, то функция $\tilde{\Phi} y \in M_{\tau \cdot \alpha}$. Таким образом, $\tilde{\Phi}\left(M_{\tau \cdot \beta}\right) \subset M_{\tau \cdot \alpha}$. Аналогично доказывается обратное включение, если в доказательстве вместо отображения $\tilde{\Phi} *$ рассмотреть отображение

$$
\left(\tilde{\Phi}^{*}\right)^{-1}: \mathbb{R}^{L_{\tau \cdot \beta}} \rightarrow \mathbb{R}^{L_{\tau \cdot \alpha}}
$$

Из предложения 1 получаем, что

$$
\begin{gathered}
M_{\tau \cdot \alpha}=\tilde{\Phi}\left(M_{\tau \cdot \beta}\right) \sim \tilde{\Phi}\left(C_{p}\left(L_{\tau \cdot \beta}\right) \times c_{0}\left(\Gamma_{\beta}\right)\right) \sim \\
\sim \tilde{\Phi}\left(C_{p}\left(L_{\tau \cdot \beta}\right)\right) \times \tilde{\Phi}\left(c_{0}\left(\Gamma_{\beta}\right)\right) \sim C_{p}\left(L_{\tau \cdot \alpha}\right) \times \tilde{\Phi}\left(c_{0}\left(\Gamma_{\beta}\right)\right) .
\end{gathered}
$$

С другой стороны,

$$
M_{\tau \cdot \alpha} \sim C_{p}\left(L_{\tau \cdot \alpha}\right) \times c_{0}\left(\Gamma_{\alpha}\right) .
$$

Отсюда, учитывая, что все дополнения к пространству $C_{p}\left(L_{\tau \cdot \alpha}\right)$ в пространстве $M_{\tau \cdot \alpha}$ являются линейно гомеоморфными, заключаем, что

$$
c_{0}\left(\Gamma_{\alpha}\right) \sim \tilde{\Phi}\left(c_{0}\left(\Gamma_{\beta}\right)\right) \sim c_{0}\left(\Gamma_{\beta}\right) .
$$

Но это невозможно [7], поскольку $|\alpha|<|\beta|$, а значит,

$$
w\left(c_{0}\left(\Gamma_{\alpha}\right)\right)=\left|\Gamma_{\alpha}\right|=\left|\omega_{\alpha}\right|<\left|\omega_{\beta}\right|=\left|\Gamma_{\beta}\right|=w\left(c_{0}\left(\Gamma_{\beta}\right)\right) .
$$

\section{ЛИТЕРАТУРА}

1. Энгелькинг Р. Общая топология. М.: Мир, 1986, 752 с.

2. Bessaga C., Pelczynski C. On isomorphic classification of spaces of continuos functions // Studia Math. 1960. V. 19. P. 53-62.

3. Semadeni Z. Banach spaces non-isomorphic to their Cartesian product // Bulletin of the Polish Academy of Sciences Ser. Math. Stron. et Phys. 1960. V. 8. P. 81-84.

4. Гулько С.П. Оськин А.В. Изоморфная классификация пространств непрерывных функций на вполне упорядоченных бикомпактах // Функциональный анализ и его приложения. 1975. Т. 9. № 1. С. 61-61.

5. Кисляков С.В. Изоморфная классификация пространств непрерывных функций на ординалах // Сиб. мат. жур. 1975. Т. 16. С. 293-300.

6. Kalenda $O$. Note on Markushevich bases in subspaces and quotients of Banach spaces // Bulletin of the Polish Academy of Sciences. Mathematics. 2002. V. 50. No. 2. P. 117-126.

7. Архангельский А.В. О линейных гомеоморфизмах пространств функций // ДАН СССР. 1982. Т. 264. № 6. С. 1289-1292.

8. Куратовский К., Мостовский А. Теория множеств. М.: Мир, 1970. 416 с. 
Trofimenko N.N. ON LINEAR HOMEOMORPHISMS OF SPACES OF CONTINUOUS FUNCTIONS ON «LONG LINES»

\section{DOI $10.17223 / 19988621 / 39 / 4$}

In this paper, we prove that for the elementary regular ordinal and arbitrary ordinals $\alpha, \beta$, $\alpha<\beta \leq \tau$, the spaces of continuous functions $C_{p}\left(L_{\tau \cdot \alpha}\right)$ and $C_{p}\left(L_{\tau \cdot \beta}\right)$, defined on the "long lines" $L_{\tau \cdot \alpha}$ and $L_{\tau \cdot \beta}$, are not linearly homeomorphic.

Keywords: «long lines», linear homeomorphisms, dual space, ordinals, initial ordinal, regular ordinal, topology of pointwise convergence, compactness.

TROFIMENKO Nadezhda Nikolaevna (Tomsk State University, Tomsk, Russian Federation)

E-mail: trofimenko@sibmail.com

\section{REFERENCES}

1. Engel'king R. Obshchaya topologiya. Moscow, Mir Publ., 1986, 752 p. (in Russian)

2. Bessaga C., Pelczynski S. On isomorphic classification of spaces of continuos functions. Studia Math., 1960, vol. 19, pp. 53-62.

3. Semadeni Z. Banach spaces non-isomorphic to their Cartesian product. Bulletin of the Polish Academy of Sciences Ser. Math. Stron. et Phys., 1960, vol. 8, pp. 81-84.

4. Gul'ko S.P. Os'kin A.V. Izomorfnaya klassifikatsiya prostranstv nepreryvnykh funktsiy na vpolne uporyadochennykh bikompaktakh. Funktsional'nyy analiz $i$ ego prilozheniya, 1975, vol. 9, no. 1, pp. 61-61. (in Russian)

5. Kislyakov S.V. Izomorfnaya klassifikatsiya prostranstv nepreryvnykh funktsiy na ordinalakh. Sib. mat. zhurn., 1975, vol. 16, pp. 293-300. (in Russian)

6. Kalenda O. Note on Markushevich bases in subspaces and quotients of Banach spaces. Bulletin of the Polish Academy of Sciences. Mathematics, 2002, vol. 50, no. 2, pp. 117-126.

7. Arkhangel'skiy A.V. O lineynykh gomeomorfizmakh prostranstv funktsiy. DAN SSSR, 1982, vol. 264, no. 6, pp. 1289-1292. (in Russian)

8. Kuratovskiy K., Mostovskiy A. Teoriya mnozhestv. Moscow, Mir Publ., 1970. 416 c. (in Russian) 